\title{
Ideenforum: Die neue Diskussion um den Sinn und Zweck von Unternehmen
}

\section{Einleitung}

Der Kapitalismus steht an einem Wendepunkt. Die Maxime des Ökonomen und Nobelpreisträgers Milton Friedman, der zufolge der 'Sinn und Zweck (Purpose) und die einzige gesellschaftliche Verantwortung von Unternehmen in der Steigerung des Profits liege (vgl. Friedman 1970), sieht sich zunehmend herausgefordert und wenngleich auch zahlreiche US-amerikanische sowie viele, insbesondere große, börsennotierte, europäische Firmen dieser Forderung seit spätestens Mitte der 1980er Jahre folgten, hat die damit verbundene, unbedingte Orientierung von Unternehmen am Shareholder Value heute ihren Zenit überschritten. Klimakrise, Altersarmut, Niedrigzinspolitik: Vor dem Hintergrund sich zuspitzender ökologischer, gesellschaftlicher und ökonomischer Probleme fordert eine breite Öffentlichkeit aus NGOs, MitarbeiterInnen, InvestorInnen und neuerdings auch global führenden UnternehmenslenkerInnen eine Neujustierung von Unternehmen und eine Hinwendung zum Stakeholder Value - darunter auch Firmenchefs, die bislang gewissermaßen als Personifikation des Kapitalismus galten (vgl. Hill 2019).

\section{Globalisierung und kurzfristige Gewinnmaximierung}

Historisch betrachtet beginnt die unbedingte Orientierung von Unternehmen an den Interessen der Shareholder zeitgleich mit der starken Globalisierungswelle, die wir in den vergangenen Dekaden beobachten konnten. Nie zuvor war es für Unternehmen einfacher, ihre Wertschöpfungsketten nach außen zu verlagern und global an Technologie-, Produktivitäts-, aber vor allem an Kostenfaktoren auszurichten. Der Erfolg lag in der Unbeirrbarkeit der Firmen: Aus der konsequent und ausschließlich profitorientierten Standortpolitik resultiert ein kurz- und mittelfristiger Ertrag in schwindelerregender Höhe. Die Gewinne waren und sind gewaltig.

Gewaltig ist allerdings auch die Skrupellosigkeit, mit der vor allem amerikanische, aber ebenso europäische Unternehmen die Globalisierung zur Maximierung dieser Profite ausnutzen. Zuerst wurden viele nationale Produktionsstätten in industriell weniger entwickelte Nachbarstaaten und später in sogenannte Billiglohnländer in Asien oder Afrika verlegt. Die daraus resultierende Arbeitslosigkeit in den Ursprungsländern sowie der Zusammenbruch gesamter ökonomischer und

* Prof. Dr. Markus Scholz, Center for Corporate Governance and Business Ethics/Fachhochschule, Währinger Straße 61, Ö-1090 Vienna, Tel. +43-(1)476-775735, E-Mail: cgbe@fh-wien.ac.at, Forschungsschwerpunkte: Verantwortung von Unternehmen, strategische Nachhaltigkeit und soziale Verantwortung von Unternehmen. 
kultureller Gemeinschaften rechneten die Unternehmen jedoch nicht ihrem eigenen Verantwortungsspektrum zu, sondern verwiesen stur auf das Argument der Arbeitsteilung von Staat und Unternehmen: Unternehmen sollen Profite maximieren, zahlen Steuern, schaffen Innovation und Arbeitsplätze. Wo sie dies tun, sei letztlich nicht relevant. Wenn sie aufgrund zu hoher Produktionskosten vom Ursprungsland in ein anderes Land wanderten, entstünden eben dort neue Arbeitsplätze, sprudelten eben dort die Steuereinahmen und könne eben dort die Infrastruktur verbessert werden. Der Standortwettbewerb erlaube es den bisher weniger industrialisierten Ländern außerdem, endlich - zumindest in ökonomischer Hinsicht - an die reichen Industrieländer aufzuschließen, da neue Märkte entstehen und von diesen fortlaufenden Prozessen letztlich alle Marktteilnehmenden profitieren würden.

Was in dieser Argumentationslinie vergessen wird: Die Attraktivität der neuen Unternehmensstandorte resultiert vor allem aus den vergleichsweise geringeren Produktionskosten. Und diese sind nicht nur den unterschiedlichen Kaufkraftniveaus geschuldet, sondern bestehen vor allem, weil die Kosten für die Einhaltung und Überwachung von sowieso schon niedrigeren Arbeits- und Umweltschutzvorschriften relativ gering sind. Wenn sich Unternehmen also aus Kostengründen dafür entscheiden, die Produktion in Billiglohnländer zu verlagern, liegt darin auch eine Entscheidung für das Gefälle dieser Schutzstandards - zumindest nehmen Firmenchefs dieses Gefälle bewusst in Kauf. In diesem Kontext wird Unternehmen vorgeworfen, die niedrigen Regulierungsniveaus bewusst auszunutzen und in ökologischer und gesellschaftlicher Hinsicht unmoralisch zu agieren. Inzwischen sind zahlreiche Beispiele weithin bekannt, in denen Unternehmen häufig gedeckt durch Sub-Unternehmen - durch die stille Akzeptanz von menschenunwürdigen Arbeitsbedingungen (vgl. Aleksynska et al. 2019) und teilweise auch durch die Duldung von Zwangs- und Sklavenarbeit Menschenrechtsverletzungen Vorschub leisten (vgl. Phung et al. 2018). Ähnlich eklatant sind die ökologischen Verfehlungen einiger Unternehmen, die sich die laschen Umweltschutzstandards ihrer Gastländer zunutze machen: Sie produzieren und entsorgen in einer Art und Weise, die in ihren Heimatländern längst untersagt sind (vgl. Greenpeace 2018). In diese Liste der vorgeblichen und realen Kostensenkungspraktiken reiht sich auch die Steuervermeidungspolitik einiger Unternehmen ein: Bekannte Internetfirmen und Mobiltelefonhersteller fertigen ihre Produkte überwiegend in Billiglohnländern, streichen ihre immensen Gewinne durch den Verkauf in Industrieländern ein und vermeiden gleichzeitig Steuern durch häufig lediglich auf dem Papier existierende Firmenkonstrukte und Adressen in Steueroasen (vgl. Preuss 2012; Remeur 2019).

\section{Die Folgen einer unbedingten Shareholderorientierung}

Wer in einer globalisierten Wirtschaft geringe oder gänzlich fehlende Arbeits- und Umweltschutzvorgaben ausnutzt oder die Steuerlast durch geschickt gewählte Unternehmenskonstruktionen verringert, agiert nicht illegal. In der Vergangenheit 
lag in solchem Verhalten von Unternehmen schlicht das probateste Mittel, um den Profit für Shareholder zu maximieren (vgl. ebd.). Getrieben von empfindlich reagierenden Finanzmarktschwankungen, mindestens vierteljährlich vorzulegenden Geschäftszahlen, einer ganzen Reihe von kurzfristig orientierten, extrem aggressiv agierenden Hedgefonds und immer aktiveren InvestorInnen standen UnternehmenslenkerInnen zunehmend unter Druck, was keineswegs apologetisch, aber doch zumindest explikativ zur Kenntnis genommen werden soll.

Die Folgen dieser Unternehmensführung, immer auf kurzfristige Profitmaximierung und das Ausnutzen aller verfügbaren Schlupflöcher ausgerichtet, werden nun in ökologischer, gesellschaftlicher und ökonomischer Hinsicht zunehmend sichtbar: Die Klimakrise ist bereits Realität, die Umweltverschmutzung erreicht gefährliche Niveaus, von der sich die Natur nicht mehr zu erholen droht, Skandale um Korruption, Menschenrechtsverletzungen in Arbeitskontexten häufen sich, die Differenzen zwischen Vorstands- und Angestelltengehältern sind eklatant und wachsen weiter. Die massiv steigende Kapitalakkumulation ist kaum mehr zu rechtfertigen. So verwundert es denn auch nicht, dass der Kapitalismus als Wirtschaftssystem weltweit an Ansehen verliert und auch die darin agierenden Vehikel, also die Unternehmen, unter Legitimierungsdruck stehen (vgl. Collier 2018; Piketty 2014; Reeves et al. 2018). Wie weit diese Legitimitätskrise bereits fortgeschritten ist, lässt sich sogar an Aussagen von Personen festmachen, welche der Kapitalismuskritik bisher unverdächtig waren. Michael Porter, Professor für Wettbewerbstheorie und strategisches Management an der Harvard Business School, konstatierte bereits vor einigen Jahren: »Capitalism is under siege. In recent years business increasingly has been viewed as a major cause of social, environmental, and economic problems. Companies are widely perceived to be prospering at the expense of the broader community « (Porter/Kramer 2011: 4). Noch weiter geht Tom Wilson, CEO der Allstate Gruppe, eines der größten Versicherungsunternehmen der Welt und Vorsitzender der US-Handelskammer: »There's no reason corporations have to exist in history. (...) To the extent we don't live up to their expectations, people can revoke those rights, levy harsher taxes, summon more regulations, or, in a bleaker scenario, change the corporate framework entirely « (Reeves et al. 2018).

\section{Die neue Diskussion um Sinn und Zweck von Unternehmen}

Vor diesem Hintergrund ist eine breite Diskussion über den Purpose, also über den Sinn und den Zweck von Unternehmen entstanden, die längst überfällig war. Kern der Debatte ist die Frage, ob Unternehmen noch andere Ziele verfolgen sollten - abseits der Profitmaximierung ihrer Shareholder. Ende August 2019 machte in diesem Kontext der Business Roundtable (BRT) Furore: Der Zusammenschluss von rund 200 prominenten WirtschaftsführerInnen großer US-amerikanischer und multinationaler Unternehmen veröffentlichte ein Statement, das eine Kehrtwende einleiten soll (vgl. Business Roundtable 2019; Kort 2019). Die Unterzeichnenden des Statements fordern nichts Geringeres als die Abkehr von der bisherigen 
Maxime. Statt einer Corporate Governance, die sich lediglich an Profitmaximierung und Shareholderinteressen ausrichtet, muss jetzt die Hinwendung zur Schaffung eines breiteren Stakeholder Values erfolgen. Konkret fordern die UnternehmenslenkerInnen mehr Menschlichkeit für die MitarbeiterInnen, in ArbeitnehmerInnen zu investieren, mit Zulieferbetrieben fair zu kooperieren, die Kommunen, in denen Unternehmen agieren, aktiv zu unterstützen und die dort lebenden Menschen zu respektieren. Für Kunden sollen Werte geschaffen werden. Im allgemein sprechen sich die UnternehmerInnen also für ein Verhalten aus, das einen langfristigen Stakeholder Value generiert (vgl. Business Roundtable 2019).

Bemerkenswert ist das BRT-Statement vor allem aus zwei Gründen: Erstens handelt es sich bei der postulierten Abkehr vom eindimensionalen Ziel der Shareholderorientierung hin zu einer multidimensionalen Stakeholderorientierung tatsächlich um einen fundamentalen Kurswechsel. Als eine der global stärksten Wirtschaftsorganisationen lobbyiert der BRT seit 1978 für das Primat der Shareholder. Die vom BRT periodisch veröffentlichten Prinzipien zur Corporate Governance betonen seither, dass Unternehmen prinzipiell und ausschließlich den Sinn und Zweck haben, ihren Shareholdern zu dienen. Dementsprechend nutzte der BRT seine immensen Ressourcen und Netzwerke in den vergangenen 40 Jahren zur Manifestierung dieses Zwecks, beispielsweise indem er stärkere Arbeitnehmerund Umweltschutzgesetze blockierte. Das neueste BRT-Statement ersetzt nun alle bisherigen und rückt, wie oben angeführt, breitere Stakeholderinteressen in den Fokus der Unternehmensführung. Vor dem Hintergrund der bisherigen Ausrichtung des BRTs ist dies revolutionär (vgl. Hill 2019).

Zweitens ist das BRT-Statement deshalb so bemerkenswert, weil die Schlagkraft der UnterzeichnerInnen als massiv zu bezeichnen ist: $\mathrm{Zu}$ ihnen zählen die CEOs

- der weltweit bedeutendsten Industrie, Handels- und Dienstleistungsunternehmen, nämlich unter anderem Jeff Bezos (Amazon), Tim Cook (Apple), James P. Hackett (Ford Motor Company), Bill McDermott (SAP), Denis E. Muilenburg (The Boeing Company), James Quincey (Coca-Cola), Chuck Robbins (Cisco Sytems, Inc.), Ginni Rometty (IBM Corporation), Michael Roman (3M), Darren W. Woods (Exxon Mobil Corporation);

- der einflussreichsten Unternehmensberatungen, beispielsweise Carmine Di Sibio (EY), Lynne M. Doughtie (KPMG), Rich Lesser (Boston Consulting Group), Bob Moritz (PWC), Punit Renjen (Deloitte), Kevin Sneader (McKinsey \& Company);

- sowie zahlreiche hochkarätige Banken, darunter Michael L. Corbat (Citigroup, Inc.) und - besonders beachtenswert - auch Großinvestoren wie Laurence Fink (Blackrock).

Vor allem die letztgenannte Gruppe der Banken und Investierenden ist in diesem Zusammenhang beachtenswert, denn hier scheint sich schon seit etwas längerer Zeit ein parallel verlaufender Trend zu entwickeln. Diese Unternehmensgruppe betont seit Beginn 2018 die hohe Relevanz einer Stakeholderorientierung, besonders in ökologischer Hinsicht. Larry Fink, CEO des global größten unabhängigen Vermögensverwalters (verwaltetes Vermögen: ca. 6 Billionen USD), betonte in sei- 
nen prominenten und einflussreichen Letters to CEOs bereits drei Jahre in Folge, dass Unternehmen ihre Rolle in der Gesellschaft, also ihren Sinn und Zweck, nicht auf reine Shareholderinteressen beschränken dürfen. Stattdessen müssen Strategien und Werkzeuge den ssocial impact s steigern, der multidimensionalen Verantwortung von Unternehmen gegenüber Land und Leuten gerecht werden (vgl. Fink 2018; 2019). Unterstützt werden solche Forderungen von weiteren Branchenriesen wie etwa der Vanguard Group (verwaltetes Vermögen: ca. 5,3 Billionen USD) und dem norwegischen staatlichen Pensionsfonds (verwaltetes Vermögen: ca. 828 Milliarden EUR).

\section{Die Neuausrichtung von Unternehmen: Substanz oder Marketing-Rhetorik?}

Hauptsächlich in Europa fragen sich RezipientInnen des BRT-Statements, wie viel Substanz hinter der Forderung nach mehr Stakeholderorientierung steckt. Tatsächlich sprechen mindestens vier Faktoren dafür, dass es sich wahrhaftig um mehr als einen leeren PR-Gag oder der reinen Marketing-Rhetorik von US-Unternehmen handelt: Erstens forcieren, wie oben bereits kurz skizziert, die global größten Vermögensverwaltungsgesellschaften und Pensionsfonds das Thema der ökologischen und gesellschaftlichen Nachhaltigkeit mit zunehmendem Interesse und rücken es damit auf die Agenden von Unternehmensvorständen. Sie fordern sie damit quasi heraus, sich der Frage nach dem Sinn und Zweck des eigenen Unternehmens neu zu stellen. Neben diesen Großinvestoren legen private AnlegerInnen ihr Geld zunehmend in nachhaltige und ethische Finanzprodukte an. Der daraus resultierende - neue - Markt wird inzwischen mit global 30,7 Billionen USD, davon 14 Billionen USD in Europa, beziffert (vgl. Chasan 2019). Institutionelle und private Investierende zwingen Unternehmensvorstände gewissermaßen dazu, dem Stakeholder Value mehr Aufmerksamkeit zu schenken und ihre Corporate Governance an langfristigeren Zielen auszurichten. Inzwischen zeigen zahlreiche empirische Studien (und inzwischen auch Metastudien), dass Gewinne auch mit Verantwortungswahrnehmung, also Unternehmenserfolg mit einer breiten Stakeholderorientierung in Korrelation stehen (vgl. Margolis et al. 2009; Orlitzky et al. 2003). Neueste Studien zeigen darüber hinaus, dass Unternehmen, die die best practices im Bereich Nachhaltigkeitsmanagement ausblenden, eine geringere Überlebenswahrscheinlichkeit besitzen (vgl. Cheng et al. 2014).

Zweitens darf davon ausgegangen werden, dass der BRT sein Statement ernst meint, da die Themen Nachhaltigkeit und Verantwortung eine immer größere Rolle für die MitarbeiterInnen spielen. MitarbeiterInnen, die sich mit den Werten des Unternehmens identifizieren und mit ihnen übereinstimmen, sind nicht nur einfacher zu attrahieren, sie sind im Arbeitsalltag auch leichter zu motivieren (vgl. Brammer et al. 2007; Glavas/Kelley 2014; Hansen et al. 2011; Kim et al. 2010; Meynhardt et al. 2018; Roeck/Delobbe 2012) und unter besonderen Bedingungen sogar bereit, auf Teile ihres Gehalts zu verzichten, sofern die entsprechende Gegenleistung von ideellem Wert zeugt (vgl. Frank/Smith 2016; Haski-Leventhal/ Concato 2016). 
Drittens reagiert der BRT mit seinem Statement möglicherweise auf politische Entwicklungen, die momentan dabei sind, die Stakeholderorientierung strenger zu formalisieren. Global existieren dazu bereits diverse zumeist freiwillige Standards und Reportingwerkzeuge, beispielsweise GRI, ISO 26000 und OECD Guidance for Responsible Business Conduct. Gerade die Europäische Union verstärkt ihre Bemühungen, Unternehmen zum ganzheitlichen Ansatz der Stakeholderorientierung, vor allem zu einer stärkeren ökologischen Orientierung, zu verpflichten. Die USA hinken auf diesem Gebiet hinterher, verpflichtende Standards sind dort bisher noch eher unbekannt, zumindest weniger ausgeprägt als in Europa. Wohingegen eine rabiatere Methode beim BRT Beunruhigung ausgelöst haben dürfte: Elisabeth Warren, US-Senatorin und zeitweise Bewerberin für die US-Präsidentschaft, will Unternehmen zur Stakeholderorientierung zwingen, ArbeitnehmerInnen zur Repräsentanz im Board of Directors drängen und der US-Regierung sogar die Möglichkeit einräumen, Unternehmen notfalls auflösen zu können (vgl. Warren 2018).

Viertens erkennen die global agierenden und bestens informierten CEOs womöglich tatsächlich die Brisanz der aktuellen weltpolitischen Lage: Die Klimakrise ist auf dem Vormarsch, die Wasser- und Ressourcenknappheit erfordern neue Produktionswege, die Bodendegradation bedroht ganze Ernten, die Vernichtung der Biodiversität stellt einen Risikofaktor für Natur und Mensch dar, die frappierende ökonomische Ungleichheit (zwischen den reichsten und den ärmsten Ländern, aber auch zwischen CEOs und Arbeitnehmenden) ist real und die Auswirkungen dieser Entwicklungen werden immer stärker spürbar. Der Anreiz für Unternehmen, die auf kurzfristige Shareholderinteressen fokussiert sind, ihren Anteil an dieser Entwicklung in relevanten Dimensionen abzuschwächen, ist bisher weder politisch noch marktgestützt gegeben. Entsprechend gering sind die Interessen dieser Unternehmen daran, Lösungen für diese globalen Herausforderungen anzubieten.

\section{Reaktionen und Konsequenzen von und für europäische Unternehmen}

Die Reaktionen europäischer, insbesonderer deutscher und österreichischer Unternehmen auf das BRT-Statement sind indes interessant $\mathrm{zu}$ beobachten. Das in Europa gewachsene und gelebte Konzept der sozialen Marktwirtschaft sowie das weit verbreitete deutsche Corporate Governance System sind - anders als der USamerikanische Shareholderkapitalismus - per se auf eine breitere Stakeholderorientierung angelegt. So sitzen ArbeitnehmervertreterInnen nicht zufällig im Aufsichtsrat von Unternehmen, sondern deren dortige verpflichtende Repräsentanz gilt als geradezu exemplarisch für die gelebte Sozialität in der Marktwirtschaft. Wenig überraschend ist daher, wie europäische UnternehmenslenkerInnen die BRT-Revolution bewerten: Christian Friege beispielsweise, der Vorstandschef des im SDax notierten Fotodienstleisters Cewe, betont die lange Tradition deutscher Unternehmen, auch soziale Verantwortung zu übernehmen: »Für mich ist an dieser Erklärung nur neu, dass sie jetzt so prominent aus den USA kommt. Inhaltlich 
entspreche sie einer Auffassung, die auch viele deutsche Mittelständler teilten « (Kort et al. 2019).

Noch eindeutiger ist die Äußerung des Familienunternehmers Ulrich Bettermann, Vorstand der Elektrotechnikherstellers Obo Bettermann: »Wenn die CEOs von 200 US-Unternehmen wegkommen wollen von der einseitigen Orientierung auf Shareholder-Value und Profitmaximierung, dann ist das sicher ein schöner Zug amerikanischer Unternehmenschefs « (ebd.). Bettermann betont weiter, dass dies in deutschen Familienunternehmen »seit Jahr und Tag Unternehmensmaxime" sei (ebd.).

Alles in bester Ordnung also? In Deutschland und Österreich - der Wiege der sozialen Marktwirtschaft - ohnehin schon immer? Können sich die UnternehmenslenkerInnen diesseits des Atlantiks beruhigt zurücklehnen und die amerikanischen KollegInnen belächeln? Diese Annahme ist aus verschiedenen Gründen gefährlich.

Auch deutsche und österreichische Unternehmen agieren nicht in einer marktfreien und von Shareholderdruck gelösten Umwelt, sondern unterliegen ebenfalls den Versuchungen der kurzfristigen Gewinnmaximierung mit all den damit verbundenen Risiken und Konsequenzen. Spektakuläre und weniger spektakuläre Unternehmensskandale wurden auch von deutschen und österreichischen Unternehmen verursacht. Exemplarisch sind hier Marktmanipulationen wie sie die Deutsche Bank und andere Banken betrieben haben (vgl. Süddeutsche Zeitung 2020), die Umweltverschmutzung großer Automobilkonzerne wie VW (vgl. The Economist 2015) oder die Korruptionsaffären von Siemens und vielen anderen europäischen Firmen (vgl. NBC News 2005). Und auch wenn die Skandale großer, börsennotierter Unternehmen die Schlagzeilen der Medien dominieren, können sich Familienunternehmen nicht per se in die weiße Weste der Unschuld hüllen. Neben den eindeutig kriminellen Machenschaften einiger großer Unternehmen nehmen auch zahlreiche Familienunternehmen die Möglichkeiten in Anspruch, die durch die Globalisierung geboten werden und verlagern ihre Produktion eben doch ganz oder teilweise in Entwicklungsländer, nutzen die dort niedrigen Umwelt-, Arbeits- und Steuerstandards sehr häufig, wie oben beschrieben, auch in Kombination mit undurchsichtigen Konstruktionen aus Sub-Unternehmen (vgl. Fröndhoff 2019).

Darüber hinaus zeigen sich zahlreiche, gerade inhabergeführte Familienunternehmen, noch sehr patriarchalisch, zuweilen auch matriarchalisch, von InhaberInnen geführt, die ihren Alleinherrscheranspruch ausleben. Diese Top-down-Führung kleidet sich häufig in den Mantel des ehrbaren Kaufmanns, dessen Lebensund Leitbild von Anstand, Respekt und Verantwortung getragen wird. Dieses von vielen FamilienunternehmerInnen bemühte Bild ist ein seit Jahrhunderten in der Individualethik beheimatetes Konzept, welches das richtige Handeln v von UnternehmerInnen in einer gegebenen Situation zum Thema hat. Die vielen Tugenden sind auch in Thomas Manns Klassiker hinreichend ausgeführt und gelobt: Der ehrbare Kaufmann ist als fleißig, ordentlich, ehrlich, sparsam, zuverlässig, demütig, gerecht und vertrauensvoll charakterisiert. Oder wie Thomas Mann es den alten Firmengründer Buddenbrook sagen ließ: »Mein Sohn, sey mit Lust bey den 
Geschäften am Tage, aber mache nur solche, dass wir bey Nacht ruhig schlafen können " (Mann 1901: 190). So verdienstvoll dies klingt - diese sympathische Leitfigur wird den drängenden Herausforderungen, welche das 21. Jahrhundert an Unternehmen stellt, kaum mehr gerecht werden können. Denn vor allem ist der ursprüngliche ehrbare Kaufmann - von Kauffrauen wird sowieso kaum geredet - ein >Unternehmer auf eigene Rechnung`, welcher persönlich haftet. Obwohl inhabergeführte Unternehmen, insbesondere in den deutschsprachigen Ländern, nach wie vor stark verbreitet sind, stellen sie heute nur eine mögliche Organisationsform wirtschaftlicher Aktivitäten dar. Das Gros der Agierenden - vom Assistenten bis zur Vorstandsvorsitzenden - nehmen als Angestellte am Wirtschaftsleben teil, sodass für sie das Leitbild des sehrbaren Kaufmanns« nur von bedingtem Interesse ist. Aber selbst die inhabergeführten Unternehmen greifen mit der Analogie des ehrbaren Kaufmanns möglicherweise zu kurz. Die Tugendethik des ehrbaren Kaufmanns setzt das Individuum ins Zentrum der Moralität - was an den komplexen, globalisierten Anforderungen unseres heutigen Wirtschaftslebens scheitern muss. Die Tugendethik des ehrbaren Kaufmanns ist zum Beispiel kaum dafür geeignet zu entscheiden, welche Stakeholder in welcher Rangfolge Priorität besitzen und für welche wann wie viel Wert geschaffen werden soll. Für den Einzelnen - auch für das moderne Individuum, das sich als extrem belastbar und weit vernetzt begreift - sind diese Entscheidungssituationen moralisch schlicht zu komplex. Der ehrenhafte Einzelkämpfer, im Sinne des ehrbaren Kaufmanns, braucht heute zusätzlich geeignete Corporate Governance Systeme, also zuverlässige Aufbau- und Ablaufstrukturen zum Wertemanagement, um die moralische Last nicht nur einem Paar Schultern aufzuladen.

Wenngleich ebenjene inhabergeführten Familienunternehmen mit all ihren Varianten des Leitbildes eines ehrbaren Kaufmanns in der Vergangenheit alle Skandale umschiffen konnten, eine gesellschaftlich, ökologisch und ökonomisch nachhaltige Unternehmenspolitik betrieben haben und deshalb zurecht als ökonomisches und moralisches Rückgrat der österreichischen und deutschen Wirtschaft gelten, stehen sie vor einer weiteren Herausforderung ungeahnter Tragweite: Ihnen bricht häufig der familiäre Nachwuchs in der Geschäftsführung weg. Und dies in doppelter Hinsicht: Einerseits beklagen viele FamilienunternehmerInnen, dass die Erbengeneration häufig kein Interesse an der Weiterführung der verantwortlichen Geschäftsführung habe. Andererseits verstehen viele FamilienunternehmerInnen, dass die Anforderungen an die Geschäftsführung ständig komplexer werden und dass bloße Familienzugehörigkeit nicht automatisch für einen Posten in der Geschäftsführung qualifiziert. Es würde demnach in jedem der zwei Fälle hervorragend ausgebildeter ManagerInnen bedürfen, um die erfolgreiche Nachfolge in der Geschäftsführung sicherzustellen. Und hier beginnt der Teufelskreis: Um die Nachfolge in Vorständen von Familienunternehmen zu sichern, wird häufig auf AbsolventInnen von MBA oder anderen betriebswirtschaftlichen Programmen zurückgegriffen, idealerweise auf Zöglinge diverser Elite-Hochschulen wie etwa der Harvard Business School, INSEAD oder Wharton. Es sind aber ebenjene Programme, welche im Verdacht stehen, in den vergangenen 20 Jahren Managergenerationen gänzlich ohne gesellschaftliches, ökologisches und ökonomisches Verant- 
wortungsbewusstsein herangezüchtet zu haben (vgl. Ghoshal 2005). Es sind genau diese von der Shareholder Value-Maximierung infizierte Generationen von ManagerInnen, welche sich nun für Vorstandsposten empfehlen - auch in Familienunternehmen.

\section{Zusammenfassung und Ausblick}

"No man is an island «, so beschreibt der amerikanische Autor John Donne in seinem gleichnamigen Gedicht eindrücklich die Vernetzung eines jeden mit der Welt: »No man is an island entire of itself; every man is a piece of the continent, a part of the main (...) « (Nutt 1999: 58). Nichts anderes gilt für Unternehmen. Kein Unternehmen ist eine Insel, sondern jedes ist vernetzt mit und beeinflusst von all seinen Stakeholdern. Negative gesellschaftliche, ökologische und ökonomische Entwicklungen führen dazu, dass relevante Stakeholder neue Anforderungen an Unternehmen stellen. Was die Sinnfrage an Unternehmen völlig neu formuliert. Die bisherige Reduktion auf die Formel Innovationen generieren, Steuern zahlen und Arbeitsplätze schaffen< ist nicht mehr ausreichend, um ein Unternehmen gesellschaftlich zu legitimieren. Ebenso wenig genügen rein philanthropische Ansätze der Unternehmensverantwortung. Emmanuel Faber, der CEO des Lebensmittelgiganten Danone, betonte dies vor Kurzem eindrücklich: »The time where I take everything and then I give back as a company or as a billionaire is over. It's good, but it's not good enough « (Hill 2019).

Der Umbruch hat jedenfalls begonnen. Er setzt sich derzeit insbesondere in den Vereinigten Staaten durch und wird vor allem von jungen ArbeitnehmerInnen, aber auch von Investierenden und nun - wie es das BRT-Statement deutlich zeigt auch von der Managerelite vorangetrieben. Europäische - auch deutsche und österreichische - Unternehmen sind gut beraten, diese Entwicklungen nicht unbeachtet zu lassen. Zwar sind die Auswirkungen eines kurzfristigen Shareholderkapitalismus in Deutschland und Österreich durch das System der sozialen Marktwirtschaft, durch das deutsche Corporate Governance System und durch vielfältige Regulierungen weniger ausgeprägt als in den USA, aber Unternehmen agieren eben auch in Europa nicht isoliert. Sie waren in der Vergangenheit ebenso in Unternehmensskandale verwickelt wie ihre amerikanischen Nachbarn, sie nutzten die aufgrund der Globalisierung und der rasant fortschreitenden technologischen Entwicklung entstandenen Regulierungslücken mitunter ebenso aus und trugen damit zum eigenen Legitimitätsverlust bei. Zudem wird der durch das BRT-Statement nun verstärkte Druck auch die europäischen Unternehmen erreichen. Es sind die im BRT organisierten Großunternehmen, die den Markt verändern werden. Die europäischen Unternehmen, welche mit diesen größeren Unternehmen mittelbar oder unmittelbar als Kunden, PartnerInnen oder Zulieferbetrieben verbunden sind, werden die Auswirkungen dieser Entwicklungen zu spüren bekommen, sei es durch erhöhte Qualitätsstandards oder strengere Berichts- und Compliance-Anforderungen. Alarmismus ist trotzdem fehl am Platz. Sollte tatsächlich eine neue Ära des Kapitalismus begonnen haben, ist sie noch jung. Die vorwie- 
gend US-amerikanischen BRT-Unternehmen müssen ihrem Postulat nach mehr Stakeholderorientierung zuerst Taten folgen lassen (vgl. The Economist 2019) und europäische Unternehmen, egal ob Familienunternehmen oder Großkonzerne, können und sollen diese Ära mitprägen. ${ }^{1}$

\section{Literaturverzeichnis}

Aleksynska, M./Berg, J./Foden, D./Johnston, H. E. S./Parent-Thirion, A./Vanderleyden, J. (2019): Working Conditions in a Global Perspective, Luxembourg, Geneva: Publications Office of the European Union, International Labor Association.

Brammer, S./Millington, A./Rayton, B. (2007): The Contribution of Corporate Social Responsibility to Organizational Commitment, in: The International Journal of Human Resource, Vol. 18/No. 10, 1701-1719.

Business Roundtable (2019): Business Roundtable Redefines the Purpose of a Corporation to Promote ran Economy that Serves All Americans<. Link: https://www.businessroundtable.o $\mathrm{rg} /$ business-roundtable-redefines-the-purpose-of-a-corporation-to-promote-an-economy-th at-serves-all-americans (last access on February 3rd, 2020).

Chasan, E. (2019): Global Sustainable Investments Rise 34 Percent to $\$ 30.7$ Trillion, in: Bloomberg, 01.04.2019. Link: https://bloomberg.com/news/articles/2019-04-01/global-sus tainable-investments-rise-34-percent-to-30-7-trillion (last access on February 3rd, 2020).

Cheng, B./Ioannou, I./Serafeim, G. (2014): Corporate Social Responsibility and Access to Finance, in: Strategic Management Journal, Vol. 35/No. 1, 1-23.

Collier, P. (2018): The Future of Capitalism: Facing the New Anxieties, London, UK: Penguin.

Fink, L. (2018): Larry Fink's 2018 Letter to CEOs. A Sense of Purpose. Link: https://www.blac krock.com/corporate/investor-relations/2018-larry-fink-ceo-letter (last access on February 3rd, 2020).

Fink, L. (2019): Larry Fink's 2019 Letter to CEOs. Purpose \& Profit. Link: https://www.blackr ock.com/corporate/investor-relations/2019-larry-fink-ceo-letter (last access on February 3rd, 2020).

Frank, D./Smith, C. (2016): Will Employees Pay to Work for a More Socially Responsible Organization? In: Academy of Management Proceedings, 13413.

Friedman, M. (1970): The Social Responsibility of Business Is to Increase Its Profits, in: The New York Times Magazine, 13.09.1970, 17.

Fröndhoff, B. (2019): Familienfirmen sind nicht automatisch bessere Unternehmen, in: Handelsblatt, 26.08.2019. Link: https://www.handelsblatt.com/meinung/kommentare/kommen tar-familienfirmen-sind-nicht-automatisch-bessere-unternehmen/24941880.html (zuletzt abgerufen am 03.02.2020).

Ghoshal, S. (2005): Bad Management Theories Are Destroying Good Management Practices, in: Academy of Management Learning \& Education, Vol. 4/No. 1, 75-91.

Glavas, A./Kelley, K. (2014): The Effects of Perceived Corporate Social Responsibility on Employee Attitudes, in: Business Ethics Quarterly, Vol. 24/No. 2, 165-202.

Greenpeace (2018): Justice for People and Planet. Link: https://www.somo.nl/wp-content/uploa ds/2018/01/Justice-for-people-and-planet.pdf (last access on February 3rd, 2020).

Hansen, S. D./Dunford, B. B./Boss, A. D./Boss, R. W./Angermeier, I. (2011): Corporate Social Responsibility and the Benefits of Employee Trust: A Cross-Disciplinary Perspective, in: Journal of Business Ethics, Vol. 102/No. 1, 29-45.

1 Ich danke Marie Czuray für die redaktionelle Unterstützung bei der Fertigstellung dieses Artikels. 
Haski-Leventhal, D./Concato, J. (2016): The State of CSR and RME in Business Schools and the Attitudes of Their Students. Third bi-annual study. Link: https://www.unprme.org/reso urce-docs/25MGSMPRMEReport2016.pdf (last access on February 3rd, 2020).

Hill, A. (2019): The Limits of The Pursuit of Profit, in: Financial Times, 23.9.2019. Link: https://ft.com/content/c998cc32-d93e-11e9-8f9b-77216ebe1f17 (last access on February 3rd, 2020).

Kim, H.-R./Lee, M./Lee, H.-T./Kim, N.-M. (2010): Corporate Social Responsibility and Employee-Company Identification, in: Journal of Business Ethics, Vol. 95/No. 4, 557569.

Kort, K. (2019): Apple und Amazon wollen sich vom Shareholder-Mantra verabschieden, in: Handelsblatt, 19.08.2019. Link: https:/handelsblatt.com/finanzen/maerkte/boerseinside/us-topmanager-apple-und-amazon-wollen-sich-vom-shareholder-mantra-verabschieden/24919706.html (zuletzt abgerufen am 03.02.2020).

Kort, K./Knitterscheidt, K./Terpitz, K./Weishaupt, G. (2019): Abschied vom Shareholder-Mantra: So reagieren deutsche CEOs auf den Brief der US-Kollegen, in: Handelsblatt, 20.08.2019. Link: https://handelsblatt.com/unternehmen/management/us-topmanager-absc hied-vom-shareholder-mantra-so-reagieren-deutsche-ceos-auf-den-brief-der-us-kollegen/24 922396.html (zuletzt abgerufen am 03.02.2020).

Mann, T. (1901): Buddenbrooks. Verfall einer Familie, Berlin: S. Fischer.

Margolis, J. D./Elfenbein, H. A./Walsh, J. P. (2009): Does It Pay to Be Good... and Does It Matter? A Meta-Analysis of the Relationship Between Corporate Social and Financial Performance. Link: https://dx.doi.org/10.2139/ssrn.1866371 (last access on February 3rd, 2020).

Meynhardt, T./Brieger, S. A./Hermann, C. (2018): Organizational Public Value and Employee Life Satisfaction: The Mediating Roles of Work Engagement and Organizational Citizenship Behavior, in: The International Journal of Human Resource Management, 1-34.

NBC News (2005): Corporate Scandals Plague Top German Firms, 08.08.2005. Link: http://w ww.nbcnews.com/id/8875874/ns/business-corporate_scandals/t/corporate-scandals-plaguetop-german-firms/ (last access on February 3rd, 2020).

Nutt, J. (1999): John Donne: The Poems. London, UK: Palgrave.

Orlitzky, M./Schmidt, F. L./Rynes, S. L. (2003): Corporate Social and Financial Performance: A Meta-Analysis, in: Organization Studies, Vol. 24/No. 3, 403-441.

Phung, K./Crane, A. (2018): The Business of Modern Slavery: Management and Organizational Perspectives, in: Clark, J. B./Poucki, S. (Eds.): The SAGE Handbook of Human Trafficking and Modern Day Slavery, London, UK: Sage, 177-197.

Piketty, T. (2014): Capital in the Twenty-First Century: A Multidimensional Approach to the History of Capital and Social Classes, in: The British Journal of Sociology, Vol. 65/No. 4, 736-747.

Porter, M. E./Kramer, M. R. (2011): Creating Shared Value: How to Reinvent Rapitalism and Unleash a Wave of Innovation and Growth, in: Harvard Business Review, Vol. 89/No. 1, 62-77.

Preuss, L. (2012): Responsibility in Paradise? The Adoption of CSR Tools By Companies Domiciled in Tax Havens, in: Journal of Business Ethics, Vol. 110/No. 1, 1-14.

Reeves, M./Dierksmeier, C./Chittaro, C. (2018): The Humanization of the Corporation. Boston Consulting Group, 08.02.2018. Link: https://bcg.com/de-at/publications/2018/humani zation-corporation.aspx (last access on February 3rd, 2020).

Remeur, C. (2019): Listing of Tax Havens By the EU. Link: http://www.europarl.europa.eu/thi nktank/en/document.html? reference=EPRS_BRI(2018)621872 (last access on February 3rd, 2020).

Roeck, K. de/Delobbe, N. (2012): Do Environmental CSR Initiatives Serve Organizations' Legitimacy in the Oil Industry? Exploring Employees' Reactions through Organizational Identification Theory, in: Journal of Business Ethics, Vol. 110/No. 4, 397-412.

Süddeutsche Zeitung (2020): Libor-Skandal. Link: https://www.sueddeutsche.de/thema/Libor-S kandal (zuletzt abgerufen am 03.02.2020). 
Taylor, P. (2018): The Dishonest Germans, in: Politico. Link: https://www.politico.eu/article/d aimler-volkswagen-bmw-porsche-audi-siemens-the-dishonest-germans/ (last access on February 3rd, 2020).

The Economist (2015): A Mucky Business, 26.09.2015. Link: https://www.economist.com/brie fing/2015/09/26/a-mucky-business (last access on February 3rd, 2020).

The Economist (2019): Scandals Suggest Standards Have Slipped in Corporate America, 06.04.2019. Link: https://www.economist.com/business/2019/04/06/scandals-suggest-stan dards-have-slipped-in-corporate-america (last access on February 3rd, 2020).

Warren, E. (2018): Warren Introduces Accountable Capitalism Act, 15.08.2018. Link: https:// www.warren.senate.gov/newsroom/press-releases/warren-introduces-accountable-capitalis m-act (last access on February 3rd, 2020). 